\title{
Cooking in the dark: a mixed reality empathy experience for the embodiment of blindness
}

\author{
RENAN GUARESE, RMIT, Australia \\ FRANKLIN BASTIDAS, UFRGS, Brazil \\ JOÃO BECKER, UFRGS, Brazil \\ MARIANE GIAMBASTIANI, UFRGS, Brazil \\ YHONATAN IQUIAPAZA, UFRGS, Brazil \\ LENNON MACEDO, UFRGS, Brazil \\ LUCIANA NEDEL, UFRGS, Brazil \\ ANDERSON MACIEL, UFRGS, Brazil \\ FABIO ZAMBETTA, RMIT, Australia \\ RON VAN SCHYNDEL, RMIT, Australia
}
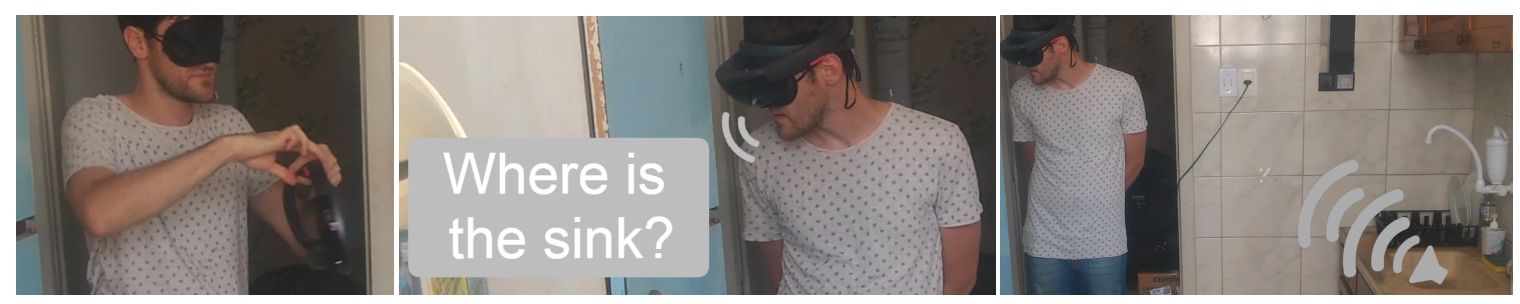

Fig. 1. Left: blindfolded user putting on the HMD. Center: user interacting with the game via voice command. Right: user receiving spatialized sound feedback.

In the context of promoting a sense of empathy for the difference in people without disabilities, we propose a gaming experience that allows users to embody having a visual impairment. By occluding the user's vision and providing spatialized audio and passive haptic feedback, allied with a speech recognition digital assistant, our goal is to offer a multi-sensory experience to enhance the user's sense of embodiment inside a mixed reality blindness simulation. Inside the game environment, while expecting a guest to arrive, the player is required to cook a meal completely in the dark. Being aided solely by their remaining senses and a digital assistant, players must go through several tasks as to prepare dinner in time, risking to lose a love interest.

Additional Key Words and Phrases: AR, Digital Empathy, Interactive Games, Embodiment, Spatial audio, Passive Haptics, HCI

\section{ACM Reference Format:}

Renan Guarese, Franklin Bastidas, João Becker, Mariane Giambastiani, Yhonatan Iquiapaza, Lennon Macedo, Luciana Nedel, Anderson Maciel, Fabio Zambetta, and Ron van Schyndel. 2021. Cooking in the dark: a mixed reality empathy experience for the embodiment of

Published in accordance with the terms of the Creative Commons Attribution 4.0 International Public License (CC BY 4.0). Permission to reproduce or distribute this work, in part or in whole, verbatim, adapted, or remixed, is granted without fee, provided that the appropriate credits are given to the original work, not implying any endorsement by the authors or by SBC.

๑ 2021 Brazilian Computing Society. 
blindness. In XR in Games Workshop, together with IMX 2021: ACM International Conference on Interactive Media Experiences. June 21-23, 2021. NY, USA. ACM, New York, NY, USA, 6 pages.

\section{INTRODUCTION}

Ever since 1989, an exhibition named Dialogue in the Dark $^{1}$ has run internationally as a visual impairment awareness experience. There, visitors face absolute darkness while performing daily activities, such as walking in a park or visiting a coffee shop in specially designed darkened rooms. More recently, researchers have claimed that the responsibility of addressing disability should be placed on everyone collectively, including technology designers [4, 22]. In that line of thought, by working together with a Visually Impaired researcher during its development and combining concepts from related projects, this paper introduces a Mixed Reality (MR) gaming experience focused on providing players with empathy towards Blind and Visually Impaired (BVI) people.

\section{RELATED WORK}

Immersive virtual environments are able to supply users with sensorimotor cues that can empower experiences with verisimilar features [1]. In order to guide user perception into experiencing situations from another person's perspective, self-representation in virtual reality has been explored and manipulated. Virtual experiences have been built that allow users to embody diverse conditions and traits, e.g. a different gender [26], nationality [11], physical stature [25], mental illness [20], and even disabilities [3, 6, 12].

Focusing on blindness simulation experiences, both commercial and academic works have been implemented recently in Virtual Reality (VR), such as an immersive documentary ${ }^{2}$, games ${ }^{3}$ and VR experiences [17][24]. In contrast to these works, the current application focuses on providing the player with a Mixed Reality approach, being set on an actual kitchen environment, with passive haptics, spatial audio localization cues and speech recognition for every interactive object in the game.

Similarly to ours, several recent studies delve into guiding users with spatial audio and sonification $[2,8,9,16]$. Among their approaches, specifically Head-Related Transfer Functions (HRTF) and Inertial Measurement Unit (IMU) sensors were also used in the current project for the synthesis of spatial sound beacons positioned according to everyday objects in the game, guiding players towards them. Despite having interesting results in their comparisons, our indoor approach targets considerable smaller dislocations in three dimensions during micro-guidance tasks in a playful game environment.

\section{GAME SCENARIO AND TASKS}

The game portrays a smart home kitchen environment. The player has a dinner date planned with a guest and the lights go out. In order to complete the game, a meal needs to be prepared and served before the guest arrives, completely in the dark. As the players do not have a previous spatial notion about the environment, digital voice assistant guides the user throughout the experience, reminding them of the recipe, cooking procedures, time constraints and how to find each required ingredient and utensil. The smart home enables sound to be projected from different objects, in a digital ventriloquism manner [16], aiding the user to find the necessary resources without any visual cues. The voice assistant is capable of speech recognition, understanding and replying to the player's questions related to the tasks. Tasks are

\footnotetext{
${ }^{1}$ History page from the Dialogue Social Enterprise website: https://www.dialogue-se.com/what-we-do/dialogue-in-the-dark/history/

${ }^{2}$ Notes On Blindness website: http://www.notesonblindness.co.uk/vr/

${ }^{3}$ Blind Game page from the Tiny Bull Studios website: https://www.tinybullstudios.com/games/blind/
} 


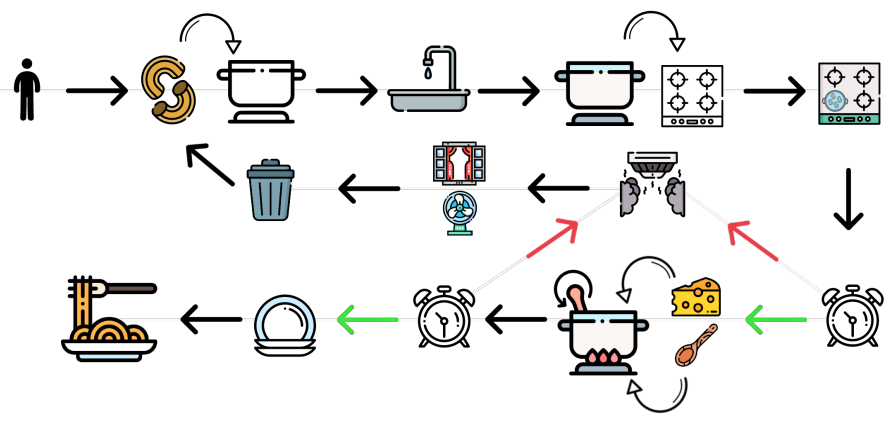

Fig. 2. Visual representation of the tasks the player has to perform in order to finish the game.

represented in Fig. $2^{4}$, which include a few time constraints that need to be obeyed, e.g. leaving the pasta too long in the boiling water will result in smoke, triggering additional tasks.

\section{MATERIALS AND METHODS}

Spatial audio. As users are required to locate objects solely on sound and passive tactile feedback, a reliable spatial audio system is necessary. As a device that already implements an approximation of HRTFs based on the current user's inter-pupilary distance coupled to inside-out head-tracking, the Microsoft HoloLens ${ }^{5}$ was chosen as the main platform for the game. As users call out for objects they need to locate, synthetic localization sounds are virtually propagated by the HoloLens from their positions, in a digital ventriloquism [16] smart-home metaphor, as shown in Fig. 1-bottom. These are meant to compensate for the lack of vision, hopefully making the player empathize with BVI people's experience and their need for assistive technologies. Besides these, several ambient sounds are also spatialized as to add to the virtual presence experience [10].

Passive haptics. Given that people might not be confident enough to make precise judgements on object locations based solely on sound, players are also expected to reach out their arms in order to understand the environment in front of them. By having real objects and props placed in the game, the user's sense of presence may be elevated [15]. Feeling the textures of, weighing and smelling ingredients are typical actions expected from cooking a meal. Thus, using the passive haptics of real kitchen utensils, wind from a fan, water from a sink and even real food [19] might arguably help the player in embodying the game protagonist.

Digital assistant. As a means to interface the smart-home elements of the game towards the virtually blind main character, a digital voice assistant was implemented. According to recent studies, people with disabilities - particularly the BVI - have been widely benefited by the use of commercially available virtual assistants in daily tasks [21]. Specifically in MR experiences, preliminary results indicated that the presence of a speech-based virtual assistant improved user performance in the execution of MR activities [23]. By taking advantage of the speech input features of the HoloLens ${ }^{6}$, voice commands related to the game tasks were implemented. Allying that to text-to-speech synthesis ${ }^{7}$, a conversational agent named Axela was created. Apart from serving as an audio interface for the game, Axela was also designed as an

\footnotetext{
${ }^{4}$ This diagram has been designed using resources from flaticon.com and freepik.com

${ }^{5}$ Audio in mixed reality page from the Microsoft website: https://docs.microsoft.com/en-us/windows/mixed-reality/design/spatial-sound

${ }^{6}$ Voice input page from the Microsoft website: https://docs.microsoft.com/en-us/windows/mixed-reality/design/voice-input

${ }^{7}$ Watson Text to Speech page from the IBM website: https://www.ibm.com/cloud/watson-text-to-speech
} 

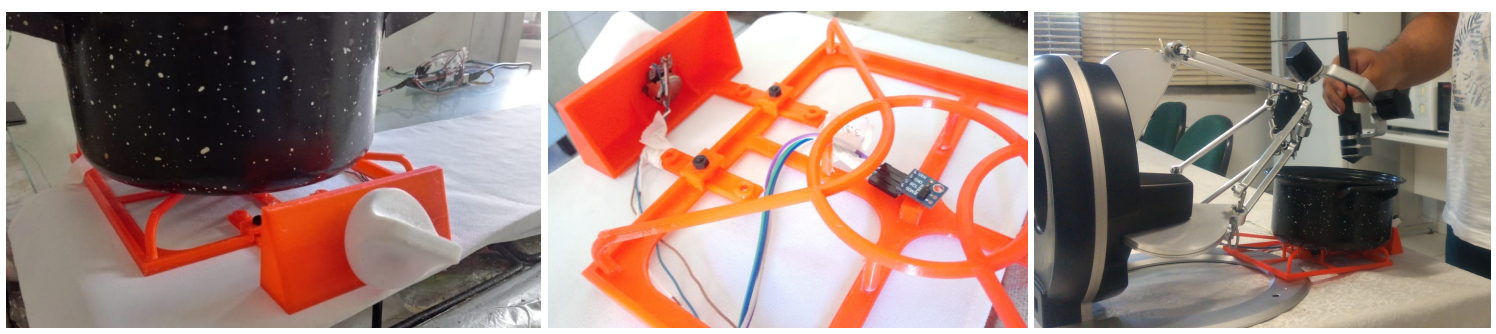

Fig. 3. Left: interactive stove knob prop, attached to potentiometer. Center: time-of-flight sensor, responsible for detecting the presence of the pot on top of the stove. Right: haptic device rendering force feedback to the user stirring the pot, virtually providing a manipulable sense of texture based on what is being cooked.

entertainment tool. The agent is capable of lightening the mood by telling sarcastic jokes, rushing the player with snark remarks when a deadline is due and setting up a background plot to motivate the entire experience.

Additional resources. Some of the interactive objects developed and 3D printed are a stove grid, a knob and a button, as shown in Fig. 3-left. These represent our stove and are used as a prop for the cooking tasks, where the grid has support for a time-of-flight sensor, depicted in Fig. 3-center, which facilitates the detection of objects on the stove. While cooking, the Omega 6 haptic device ${ }^{8}$ is used to simulate cutlery such as spoons and forks. This provides a series of realistic haptic characteristics like the change of viscosity of a fluid according to cooking time and mixing speed, in addition to feeling the collision of virtual objects inside the pot by kinesthetic stimuli [13]. All of this is exemplified in Fig. 3-right, showing user interaction stirring a pot without the need for every physical ingredient to be present, since their vision is already occluded.

\section{CONCLUSIONS AND FUTURE WORK}

The game project meant for the Microsoft HoloLens (1st gen) in its entirety is available online to be tested ${ }^{9}$. A video demonstration is also available ${ }^{10}$. The game can be played in three different backend modes: fully automated - granted the availability of the additional hardware required -; wizard-of-oz - requiring a second person to moderate the game by controlling a game server; - and standalone - entirely by yourself, using voice commands to ask Axela for instructions (e.g. "Axela, talk to me", "Axela, next"). Despite the chosen mode, a moderator is still required to place the real objects (e.g. the cooking pot, the pasta, the cheese) around the kitchen environment and pinpoint them using the HoloLens.

Our prototype pushes the state-of-the-art in Mixed Reality by focusing a gaming experience towards BVI people, making use of an innovative interface, solely composed of voice commands, spatial audio and tactile feedback. It also includes users without visual impairments in an empathy experience for the embodiment of blindness. Once it is safe to perform broad in loco user tests according to COVID-19 restrictions, both BVI and sighted users will be invited to test the game. The proposal is to evaluate its usability, cognitive and physical demands based on standardized tests $[5,14,18]$. Besides that, an empathy measurement questionnaire based on a previous work [7] will be applied to sighted users before and after the experiments. The goal is to assess how their views regarding BVI people's experiences are affected by interacting with the game.

\footnotetext{
${ }^{8}$ Products page from the Force Dimension website: https://www.forcedimension.com/products

${ }^{9}$ Cooking in the Dark repository: https://bitbucket.org/renghp/ppgc/src/blindnessGame/

${ }^{10}$ Cooking in the Dark video demonstration: https://youtu.be/GIkhCriA-3w
} 


\section{REFERENCES}

[1] Sun Joo Ahn. 2011. Embodied experiences in immersive virtual environments: Effects on pro-environmental attitude and behavior. Ph.D. Dissertation. Citeseer.

[2] Robert Albrecht, Riitta Väänänen, and Tapio Lokki. 2016. Guided by Music: Pedestrian and Cyclist Navigation with Route and Beacon Guidance. Personal Ubiquitous Comput. 20, 1 (Feb. 2016), 121-145. https://doi.org/10.1007/s00779-016-0906-z

[3] Daniel Bacchus. 2019. Life is Beautiful. Always: Using virtual reality to share the experience of disability. (2019).

[4] Stacy M. Branham and Shaun K. Kane. 2015. Collaborative Accessibility: How Blind and Sighted Companions Co-Create Accessible Home Spaces. In Proceedings of the 33rd Annual ACM Conference on Human Factors in Computing Systems (Seoul, Republic of Korea) (CHI '15). Association for Computing Machinery, New York, NY, USA, 2373-2382. https://doi.org/10.1145/2702123.2702511

[5] John Brooke. 1996. SUS: A quick and dirty usability scale.

[6] Aimee Calepso, Renan Guarese, Rafael Valer, Yhonatan Jesus Iquiapaza Ccama, Victor Adriel Oliveira, Luciana Nedel, and Anderson Maciel. 2020. 3DUI and the Phantom Limb: multisensory experience for embodiment of amputation. In 2020 IEEE Conference on Virtual Reality and 3D User Interfaces Abstracts and Workshops (VRW). 517-518. https://doi.org/10.1109/VRW50115.2020.00107

[7] Jennifer Edson Escalas and Barbara B. Stern. 2003. Sympathy and Empathy: Emotional Responses to Advertising Dramas. Fournal of Consumer Research 29, 4 (03 2003), 566-578. https://doi.org/10.1086/346251 arXiv:https://academic.oup.com/jcr/article-pdf/29/4/566/17927772/29-4-566.pdf

[8] Sylvain Ferrand, François Alouges, and Matthieu Aussal. 2017. Binaural spatialization methods for indoor navigation. In Audio Engineering Society Convention 142. Berlin, Germany. https://hal.archives-ouvertes.fr/hal-02948560

[9] Sylvain Ferrand, Francois Alouges, and Matthieu Aussal. 2018. An Augmented Reality Audio Device Helping Blind People Navigation. In Computers Helping People with Special Needs, Klaus Miesenberger and Georgios Kouroupetroglou (Eds.). Springer International Publishing, Cham, 28-35.

[10] Borko Furht (Ed.). 2008. Virtual Presence. Springer US, Boston, MA, 967-968. https://doi.org/10.1007/978-0-387-78414-4_254

[11] Victoria Groom, Jeremy N Bailenson, and Clifford Nass. 2009. The influence of racial embodiment on racial bias in immersive virtual environments. Social Influence 4, 3 (2009), 231-248.

[12] R. Guarese, V. d. J. Oliveira, A. Calepso, , R. Valer, Y. Iquiapaza, L. Nedel, and A. Maciel. 2020. E-mpathy and the Phantom Limb Sensation: a multisensory experience for embodiment of amputation. In Proceedings of the XChange Reality! 2020 St. Pölten, Austria, April 27-30, 2020. 13-16. http://ceur-ws.org/Vol-2618/

[13] Blake Hannaford and Allison M Okamura. 2016. Haptics. Springer International Publishing, Cham, 1063-1084. https://doi.org/10.1007/978-3-31932552-1_42

[14] Sandra G. Hart and Lowell E. Staveland. 1988. Development of NASA-TLX (Task Load Index): Results of Empirical and Theoretical Research. In Human Mental Workload, Peter A. Hancock and Najmedin Meshkati (Eds.). Advances in Psychology, Vol. 52. North-Holland, 139 - 183. https: //doi.org/10.1016/S0166-4115(08)62386-9

[15] Brent Edward Insko. 2001. Passive Haptics Significantly Enhances Virtual Environments. Ph.D. Dissertation. Advisor(s) Brooks, Frederick P. AAI3007820.

[16] Yasha Iravantchi, Mayank Goel, and Chris Harrison. 2020. Digital Ventriloquism: Giving Voice to Everyday Objects. In Proceedings of the 2020 CHI Conference on Human Factors in Computing Systems (Honolulu, HI, USA) (CHI '20). Association for Computing Machinery, New York, NY, USA, 1-10. https://doi.org/10.1145/3313831.3376503

[17] Redouane Kachach, Pablo Perez, Alvaro Villegas, and Ester Gonzalez-Sosa. 2020. Blindness Visualizer: A Simulated Navigation Experience. Proceedings - 2020 IEEE Conference on Virtual Reality and 3D User Interfaces, VRW 2020, 504-506. https://doi.org/10.1109/VRW50115.2020.00107

[18] Robert S. Kennedy, Norman E. Lane, Kevin S. Berbaum, and Michael G. Lilienthal. 1993. Simulator Sickness Questionnaire: An Enhanced Method for Quantifying Simulator Sickness. The International fournal of Aviation Psychology 3, 3 (1993), 203-220. https://doi.org/10.1207/s15327108ijap0303_3

[19] Rohit Ashok Khot and Florian Mueller. 2019. Human-Food Interaction. Foundations and Trends® in Human-Computer Interaction 12, 4 (2019), 238-415. https://doi.org/10.1561/1100000074

[20] David L Penn, James D Ivory, Abigail Judge, et al. 2010. The virtual doppelganger: Effects of a virtual reality simulator on perceptions of schizophrenia. The fournal of nervous and mental disease 198, 6 (2010), 437-443.

[21] Kevin M. Storer, Tejinder K. Judge, and Stacy M. Branham. 2020. "All in the Same Boat": Tradeoffs of Voice Assistant Ownership for Mixed-VisualAbility Families. Conference on Human Factors in Computing Systems - Proceedings. https://doi.org/10.1145/3313831.3376225

[22] Anja Thieme, Cynthia L. Bennett, Cecily Morrison, Edward Cutrell, and Alex S. Taylor. 2018. "I can do everything but see!" - How people with vision impairments negotiate their abilities in social contexts. Conference on Human Factors in Computing Systems - Proceedings 2018-April. https://doi.org/10.1145/3173574.3173777

[23] Francesco Vona, Emanuele Torelli, Eleonora Beccaluva, and Franca Garzotto. 2020. Exploring the Potential of Speech-based Virtual Assistants in Mixed Reality Applications for People with Cognitive Disabilities. Proceedings of the International Conference on Advanced Visual Interfaces, 1-9. https://doi.org/10.1145/3399715.3399845

[24] Zhiquan Wang, Huimin Liu, Yucong Pan, and Christos Mousas. 2020. Experiencing and Navigating Virtual Reality without Sight. Proceedings - 2020 IEEE Conference on Virtual Reality and 3D User Interfaces, VRW 2020, 519-520. https://doi.org/10.1109/VRW50115.2020.00114

[25] Nick Yee and Jeremy N Bailenson. 2007. The Proteus effect: Self transformations in virtual reality. Human Communication Research 33, 3 (2007), $271-90$. 
[26] Nick Yee, Nicolas Ducheneaut, Mike Yao, and Les Nelson. 2011. Do men heal more when in drag? Conflicting identity cues between user and avatar. In Proceedings of the SIGCHI conference on Human factors in computing systems. 773-776. 